\title{
Effects of two Lactobacillus strains on lipid metabolism and intestinal microflora in rats fed a high-cholesterol diet
}

\author{
Ning Xie ${ }^{1}$, Yi Cui ${ }^{1}$, Ya-Ni Yin ${ }^{1}$, Xin Zhao ${ }^{1}$, Jun-Wen Yang ${ }^{1}$, Zheng-Gen Wang ${ }^{2}$, Nian Fu ${ }^{3}$, Yong Tang ${ }^{4}$, \\ Xue-Hong Wang ${ }^{1}$, Xiao-Wei Liu', Chun-Lian Wang ${ }^{1}$ and Fang-Gen Lu ${ }^{1 *}$
}

\begin{abstract}
Background: The hypocholesterolemic effects of lactic acid bacteria ( $L A B)$ have now become an area of great interest and controversy for many scientists. In this study, we evaluated the effects of Lactobacillus plantarum 9-41A and Lactobacillus fermentum M1-16 on body weight, lipid metabolism and intestinal microflora of rats fed a highcholesterol diet.

Methods: Forty rats were assigned to four groups and fed either a normal or a high-cholesterol diet. The LABtreated groups received the high-cholesterol diet supplemented with Lactobacillus plantarum 9-41-A or Lactobacillus fermentum M1-16. The rats were sacrificed after a 6-week feeding period. Body weights, visceral organ and fat pad weights, serum and liver cholesterol and lipid levels, and fecal cholesterol and bile acid concentrations were measured. Liver lipid deposition and adipocyte size were evaluated histologically.

Results: Compared with rats fed a high-cholesterol diet but without LAB supplementation, serum total cholesterol, low-density lipoprotein cholesterol and triglycerides levels were significantly decreased in LAB-treated rats $(p<$ 0.05), with no significant change in high-density lipoprotein cholesterol levels. Hepatic cholesterol and triglyceride levels and liver lipid deposition were significantly decreased in the LAB-treated groups $(p<0.05)$. Accordingly, both fecal cholesterol and bile acids levels were significantly increased after LAB administration $(p<0.05)$. Intestinal Lactobacillus and Bifidobacterium colonies were increased while Escherichia coli colonies were decreased in the LABtreated groups. Fecal water content was higher in the LAB-treated groups. Compared with rats fed a highcholesterol diet, administration of Lactobacillus plantarum 9-41-A resulted in decreases in the body weight gain, liver and fat pad weight, and adipocytes size $(p<0.05)$.

Conclusions: This study suggests that $L A B$ supplementation has hypocholesterolemic effects in rats fed a highcholesterol diet. The ability to lower serum cholesterol varies among LAB strains. Our strains might be able to improve the intestinal microbial balance and potentially improve intestinal transit time. Although the mechanism is largely unknown, L. plantarum 9-41-A may play a role in fat metabolism.
\end{abstract}

\section{Background}

Elevated serum cholesterol level is widely recognized as a contributory risk factor for the development of cardiovascular diseases (CVD) such as atherosclerosis, coronary heart disease and stroke. The World Health Organization (WHO) has predicted that by 2030, CVD will remain the

\footnotetext{
* Correspondence: lufanggenyao@163.com

'Department of Gastroenterology, Xiangya Second Hospital, Central South

University, Changsha 410011, Hunan Province, PR China

Full list of author information is available at the end of the article
}

leading causes of death and affect approximately 23.6 million people globally [1]. It has been reported that even a $1 \%$ reduction in serum cholesterol could reduce the risk of coronary heart disease by 2-3\% [2]. Current drug therapies, with their high relative costs and associated side effects, are not viewed to be the optimal long-term answers. The development of alternative management strategies for the treatment of hypercholesterolemia is necessary, especially for people with borderline cholesterol levels.

\section{Biomed Central}

(c) 2011 Xie et al; licensee BioMed Central Ltd. This is an Open Access article distributed under the terms of the Creative Commons Attribution License (http://creativecommons.org/licenses/by/2.0), which permits unrestricted use, distribution, and reproduction in any medium, provided the original work is properly cited. 
Since Mann and Spoerry [3] discovered the hypocholesterolemic effects of fermented milk ingested by the Massai tribes people, the relationship between lactic acid bacteria (LAB) and the serum cholesterol has become a focus of great interest. Studies evaluating this relationship have found that lactobacilli or bifidobacteria can exhibit hypocholesterolemic properties in animal models [4-6] and in humans [7-10]. Several hypotheses have been proposed to explain these findings: (1) consumption of cholesterol by intestinal bacteria, thus reducing the amount of cholesterol available for absorption [11,12]; (2) cholesterol may be bound to the bacterial cellular surface [13] or incorporated into the bacterial cellular membranes [14] or converted into coprostanol by cholesterol reductase, which is produced by strains of lactobacilli [15]; (3) inhibition of micelle formation by certain probiotic strains [16]; (4) short-chain fatty acids produced upon selective fermentation of food by intestinal bacterial microflora may lower plasma cholesterol levels [17]; and (5) some bacterial species excrete bile salt hydrolase, leading to increased bile excretion in feces [18]. However, other reports are contradictory and fail to show hypocholesterolemic effects of probiotics $[19,20]$. Consequently, this area remains controversial. Therefore, more information is required to strengthen the proposed hypotheses and improve our undetstanding of how bacteria affect cholesterol metabolism, which might lead to more appropriate use of probiotics.

To date, most investigations on the effects of LAB on cholesterol have focused on species isolated from dairy products or feces of infants. In our previous work, we isolated several strains of lactobacilli from the stool of healthy adult Chinese volunteers. In in vitro screening-experiments, more than 30 strains of the bacteria demostrated a cholesterol-lowering activity. Among these strains, two strains, 941A and M1-16 expressed the highest cholesterol-removing abilities in their growth medium and were selected for the present study. We aimed to: 1) examine the effects of these two strains on cholesterol levels, body weight and intestinal microflora in rats; and 2) explore the possible mechanisms by which these strains might exert their hypocholesterolemic effects.

\section{Methods}

\section{Preparation of bacterial cultures}

The two strains used in this study, Lactobacillus plantarum 9-41-A [GenBank accession no. HQ424577] and Lactobacillus fermentum M1-16 [GenBank accession no. HQ423153], were two local strains isolated from the feces of healthy adults and stored at $-80^{\circ} \mathrm{C}$. They showed good survival at low $\mathrm{pH}$, tolerance to high bile concentration, and abilities to lower cholesterol in in vitro trials. After recovery at $35^{\circ} \mathrm{C}$ for 30 minutes in a biochemical incubator, the two strains were separately inoculated into
MRS liquid broth and placed in an anaerobic workstation at $35^{\circ} \mathrm{C}$ for 24 hours. The strains were harvested by centrifugation at $2000 \times \mathrm{g}$ for 20 minutes, washed twice with normal saline $(0.9 \% \mathrm{NaCl})$, and resuspended at $2 \times 10^{9}$ $\mathrm{CFU} / \mathrm{mL}$ in sterile normal saline. Subsequently, $2 \mathrm{~mL}$ of the solution was administered intragastrically to the rats daily.

\section{Animal groups and diets}

Forty male Sprague-Dawley rats (conventional clean animal grade), aged 5 weeks and weighing $120.2 \pm 4.5$ grams, were purchased from Slaccas Laboratory Animal Ltd. (Shanghai, China). All rats were housed individually in metal cages under a controlled room temperatures (20 \pm $2{ }^{\circ} \mathrm{C}$ ) and humidity (50 $\left.\pm 5 \%\right)$, and maintained on a constant 12-hour/12-hour light/dark cycle. The care and use of the animals followed our institutional and national guidelines and all experimental procedures involving animals were approved by the Ethics Committee of the Central South University.

A 1-week adaptive period on a normal diet containing $32 \%$ (weight/weight) protein, $5 \%$ fat, $2 \%$ fiber, $1.8 \%$ calcium, $1.2 \%$ phosphorus, and a nitrogen-free extract as the remainder (Commercial Chow, Kangqiao Inc., Beijing, China) was instituted [21]. The 40 rats were randomly selected and assigned to four groups of 10 rats each. The initial average body weight was similar among the four groups. The four groups were assigned diets according to the following regimen: (1) control group, normal diet; (2) model group, high-cholesterol diet; (3) L.9-41-A group, high-cholesterol diet + L. plantarum 9-41-A; (4) L.M1-16 group, high-cholesterol diet + L. fermentum M1-16. The high-cholesterol diet contained $1 \%$ (weight/weight) cholesterol, $10 \%$ lard, $10 \%$ sucrose, $0.3 \%$ sodium cholate, $0.2 \%$ propylthiouracil and a commercial chow mix (Aoboxing Biotech Co., Ltd., Beijing, China) [21]. Rats had free access to water and their group-specific diet. The L.9-41- $A$ group and L.M1-16 groups received $2 \mathrm{~mL}\left(10^{9} \mathrm{CFU} / \mathrm{mL}\right)$ daily of L. plantarum 9-41-A and L. fermentum M1-16 solutions respectively, intragastrically for the 6-week study period. Control and model groups received an equivalent amount of normal saline. Body weight was recorded weekly and food consumption was monitored daily. After the feeding period, the rats were fasted for 12 hours and euthanized. The weight of visceral organs (liver, spleen, and kidney) and mesenteric, perirenal, and epididymal white adipose tissues (WAT) were measured.

\section{Assay for serum lipids}

Blood sample $(4 \mathrm{~mL})$ was obtained from the celiac vein and transferred to nonheparinized vacuum collection tubes. Tubes were initially held stationary at $0^{\circ} \mathrm{C}$ for 30 minutes, and then centrifuged at $2000 \times \mathrm{g}$ for 15 minutes at $4^{\circ} \mathrm{C}$. Serum total cholesterol $(\mathrm{TCH})$, high-density 
lipoprotein cholesterol (HDL-C), low-density lipoprotein cholesterol (LDL-C) and triglycerides (TG) were measured with commercial kits (Daiichi Chemicals Co., Ltd, Tokyo, Japan) and a chemical analyzer 7020 (Hitachi, Tokyo, Japan). Atherogenic indexes (TCH/HDL-C and TG/HDL$\mathrm{C}$ ratios) were then calculated.

\section{Assay for liver TCH, TG and fecal sterol contents}

After euthanasia, rat livers were removed, rinsed with physiological saline solution, blotted dry and weighed. The middle lobe was standardized as the sampling region. A portion of the tissues was sectioned and soaked in 10\% (volume/volume) formaldehyde for 24 hours for subsequent staining, with the remainder stored at $-80^{\circ} \mathrm{C}$ for further testing. Liver $\mathrm{TCH}$ and $\mathrm{TG}$ contents were determined after homogenization with Folch solution(chloroform/methanol ratio $=2: 1$ ) [22] .

Fecal droppings were collected during the last 3 days of life, and fecal neutral and acidic sterols were extracted using Tokunaga' s method [23]. A sample (1 gram) of wet feces was extracted with $10 \mathrm{~mL}$ of acetone/ethanol (1:1) mixture. After drying, $3 \mathrm{~mL}$ of diethyl ether and $3 \mathrm{~mL}$ of $1 \mathrm{~N} \mathrm{NaOH}$ solution were added to separate the neutral and acidic sterols. The ether phase containing neutral sterols was dried and dissolved in $3 \mathrm{~mL}$ of acetic acid for cholesterol measurement. The $\mathrm{NaOH}$ solution phase was used for bile acid measurement with a commercial kit (Daiichi Chemicals Co., Ltd., Tokyo, Japan).

\section{Histopathology of liver and adipocytes in the WAT}

Liver samples from each rat were fixed in 10\% (volume/ volume) formaldehyde, embedded in paraffin and stained with hematoxylin-eosin (HE). The perirenal WAT was removed and embedded after delipidation. After fixation in $20 \%(\mathrm{v} / \mathrm{v})$ formalin/1\% glutaraldehyde, 3-mm tissue sections were created and stained with HE [24]. We took three samples from liver and the WAT of each rat, and made three replicates for each sample. All histologic section was observed under 5 high-power fields. The classification and degree of fatty deposition used was as follows [22]: +, mild fatty degeneration, fatty hepatocytes occupy $30 \%-50 \%$ of the hepatic parenchyma; ++ , moderate fatty degeneration, fatty hepatocytes occupy $50 \%-75 \%$ of the hepatic parenchyma; +++ , severe fatty degeneration fatty hepatocytes occupy $>75 \%$ of the hepatic parenchyma. The number and size of the adipocytes in the WAT were measured using an ocular micrometer (YanFeng Instrument Co., Ltd., Hengyang, China).

\section{Analysis of intestinal microflora}

The cecum and a portion of the adjacent colon tissue of each rat were removed and placed in capped sterile tubes. After transfer to a laminar flow cabinet, 1 gram of each sample was transferred to a tube with $9 \mathrm{~mL}$ of
$0.9 \% \mathrm{NaCl}$ solution and homogenized by vortexing for 10 minutes. Ten fold serial dilutions of each sample were performed to obtain $10-\mu \mathrm{L}$ concentrations, which were plated with selective culture medium in triplicate. EMB agar $(\mathrm{pH} 7.1 \pm 0.2)$ was used for Escherichia coli (E. coli), TCH sodium azide agar $(\mathrm{pH} 7.3 \pm 0.1)$ was used for Enterococcus faecalis, LBS agar ( $\mathrm{pH} 5.5 \pm 0.2)$ was used for LAB, and BS agar $(\mathrm{pH} 7.2 \pm 0.1)$ was used for bifidobacteria. All media were obtained from Hope Bio-Technology (Qingdao, China). Aerobic plates were placed in a biochemistry incubator at $37^{\circ} \mathrm{C}$ for 24 hours while anaerobic plates incubated at $37^{\circ} \mathrm{C}$ for 48 hours. The colonies counted after incubation represented the numbers of E. coli, Enterococcus faecalis, Lactobacillus, and Bifidobacterium.

\section{Measurement of fecal water content}

In the middle of the third and sixth weeks, rat feces were collected, weighed and dried at $80^{\circ} \mathrm{C}$ in a DZF-6050 vacuum drying oven (MingKe Instrument Co., Ltd., Shengzhen, China). until a constant weight was achieved within 24 hours, and then reweighed. Fecal water content was calculated as follows: fecal water content (\%) = [(weight before drying-weight after drying)/weight before drying] $\times 100$.

\section{Statistical Analysis}

All data was analysed in duplicate with SPSS 15.0 software (SPSS Inc., Chicago, IL, USA). One-way analysis of variance (ANOVA) with Duncan's multiple range test was performed to compare any significant differences $(\mathrm{p}<$ 0.05 ) in the variables between groups. Experimental data were presented as the mean \pm standard deviations (SD) of the mean. Rank and frequency data were analyzed by a nonparametric test.

\section{Results}

\section{Growth of rats}

All the rats appeared healthy throughout the feeding period. There were no significant differences in total food intake ( $p>0.05$ ) among the four groups. All rats fed the high-cholesterol diet exhibited higher $(\mathrm{p}<0.05)$ body weight gains and food efficiencies than control rats (Table 1). The L.9-41-A group showed less weight gain (a reduction of $13.3 \%, \mathrm{p}<0.05$ ) and food efficiency than the model group while the L.M1-16 group had no obvious difference in weight gain and food efficiency compared with the model group.

\section{Blood lipid analyses}

Serum TCH, HDL-C, LDL-C and TG levels in the four groups were shown in Figure 1A. The TCH and LDL-C levels differed significantly among the four groups. Rats fed a high-cholesterol diet had greatly increased levels of 
Table 1 Body weight gain, total food intake, and food efficiency ( $n=10$ per group) after 6 weeks

\begin{tabular}{cccc}
\hline Group & Body weight gain $(\mathbf{g})$ & Total food intake $(\mathbf{g})$ & Food efficiency*(\%) \\
\hline Control & $144.6^{\mathrm{c}} \pm 10.2$ & $879.9^{\mathrm{a}} \pm 18.9$ & $16.4^{\mathrm{c}} \pm 1.1^{\mathrm{a}}$ \\
Model & $177.0^{\mathrm{a}} \pm 8.8$ & $875.1^{\mathrm{a}} \pm 25.6$ & $20.2^{\mathrm{a}} \pm 1.0$ \\
L. 9-41-A & $153.5^{\mathrm{b}} \pm 8.2$ & $881.3^{\mathrm{a}} \pm 21.2$ & $17.4^{\mathrm{b}} \pm 1.0$ \\
L.M1-16 & $172.4^{\mathrm{a}} \pm 10.4$ & $883.9^{\mathrm{a}} \pm 22.7$ & $19.50^{\mathrm{a}} \pm 1.0$ \\
\hline
\end{tabular}

Control group: normal diet; model group: high-cholesterol diet; L.9-41-A group: high-cholesterol diet + L. plantarum 9-41-A; L.M1-16 group: high-cholesterol diet+ L. fermentum M1-16.

${ }^{*}$ Food efficiency $(\%)=$ (body weight gain/food intake) $\times 100$.

The data are shown as the mean \pm standard deviation ${ }^{a, b, c}$ Mean values within a column with different superscript letters differ significantly $(\mathrm{p}<0.05, \mathrm{n}=10$ ).

serum TCH, LDL-C and TG levels compared with rats fed the normal diet $(\mathrm{p}<0.05)$. Compared with the control group, the model group showed a $63.7 \%$ increase in $\mathrm{TCH}(254.89 \pm 22.47$ vs. $155.72 \pm 15.96 \mathrm{mg} / \mathrm{dL}, \mathrm{p}<$ $0.05)$, a $176.6 \%$ increase in LDL-C (155.16 $\pm 22.43 v s$. $56.10 \pm 14.03 \mathrm{mg} / \mathrm{dl}, \mathrm{p}<0.05)$ and a $60.3 \%$ increase in TG $(128.31 \pm 12.05$ vs. $80.03 \pm 12.39 \mathrm{mg} / \mathrm{dL}, \mathrm{p}<0.05)$. The two LAB strains displayed in vivo hypocholesterolemic abilities. Compared with the model group, the L.941-A group achieved a maximal $\mathrm{TCH}$ reduction of $25.3 \%(190.36 \pm 20.67$ vs. $254.89 \pm 22.47 \mathrm{mg} / \mathrm{dL}, \mathrm{p}<$ $0.05)$ and an LDL-C reduction of $32.9 \%(104.13 \pm 16.11$ vs. $155.16 \pm 22.43 \mathrm{mg} / \mathrm{dL}, \mathrm{p}<0.05)$ while the $L . \mathrm{M} 1-16$ group achieved a TCH reduction of $12.5 \%$ (223.12 \pm $16.84 v s .254 .89 \pm 22.47 \mathrm{mg} / \mathrm{dL}, \mathrm{p}<0.05)$ and an LDL$\mathrm{C}$ reduction of $17.3 \%(128.28 \pm 15.43 \mathrm{mg} / \mathrm{dL}$ vs. 155.16 $\pm 22.43 \mathrm{mg} / \mathrm{dL}, \mathrm{p}<0.05)$. The control group showed the highest HDL-C concentration, and the two LAB strains did not demonstrate obvious influences on the HDL-C levels. However, serum TG level was significantly lower in the LAB-treated groups than in the model group. Specifically, the L.9-41-A group displayed a TG reduction of $16.9 \%(106.65 \pm 12.58$ vs. $128.31 \pm$ $12.05 \mathrm{mg} / \mathrm{dL}, \mathrm{p}<0.05)$ while the L.M1-16 group displayed a TG reduction of $15.7 \%(108.12 \pm 13.35 \mathrm{vs}$. $128.31 \pm 12.05 \mathrm{mg} / \mathrm{dL}, \mathrm{p}<0.05)$, while the two LABtreated groups showed no significant difference $(\mathrm{p}>$ $0.05)$. The control group had the lowest $(\mathrm{p}<0.05)$ atherogenic indexes among all four groups. Compared with the model group, the atherogenic indexes were significantly lower in the L.9-41-A group (TCH/HDL-C ratio, $2.95 \pm 0.54$ vs. $4.08 \pm 0.57$; TG/HDL-C ratio, 1.65 \pm 0.30 vs. $2.06 \pm 0.29$ ) and the L.M1-16 group (TCH/ HDL-C ratio $3.40 \pm 0.36$ vs. $4.08 \pm 0.57$; TG/HDL-C ratio, $1.66 \pm 0.32$ vs. $2.06 \pm 0.29$ ) (Figure 1B). Between the two LAB-treated groups, the L.9-41-A group had lower $(\mathrm{p}<0.05)$ TCH/HDL-C ratio than the L.M1-16 group, and the TG/HDL-C ratio was similar between the two groups ( $\mathrm{p}>0.05)$.

\section{Liver and fecal lipid analyses}

Liver and fecal lipid contents were shown in Figures 1C and 1D. The control group displayed the lowest levels of both hepatic TCH $(10.69 \pm 1.54 \mathrm{mg} / \mathrm{g})$ and TG $(17.90 \pm$ $2.67 \mathrm{mg} / \mathrm{g}$ ). The L.9-41-A and L.M1-16 groups showed no obvious differences in both hepatic TCH and TG levels, but they all had lower concentrations $(\mathrm{p}<0.05)$ than the model group $(\mathrm{TCH}, 14.70 \pm 1.42$ and $14.82 \pm$ 1.31 vs. $18.93 \pm 1.59 \mathrm{mg} / \mathrm{g}$, respectively; TG, $25.82 \pm$ 2.50 and $26.44 \pm 2.68 \mathrm{vs} .33 .14 \pm 2.19 \mathrm{mg} / \mathrm{g}$, respectively). The high-cholesterol diet increased the fecal excretion of cholesterol and bile acids. Compared with the model group, the L.9-41-A group displayed a $39.2 \%$ increase in fecal cholesterol $(39.94 \pm 2.39$ vs. $28.68 \pm$ $3.48 \mathrm{mg} /$ day, $\mathrm{p}<0.05)$ and a $40 \%$ increase in fecal bile acids $(22.70 \pm 2.29 v s .16 .21 \pm 3.68 \mathrm{mg} /$ day, $\mathrm{p}<0.05)$ while the L.M1-16 group showed a $30.2 \%$ increase in fecal cholesterol $(37.33 \pm 2.92$ vs. $28.68 \pm 3.48 \mathrm{mg} /$ day, $\mathrm{p}<0.05)$ and a $19 \%$ increase in fecal bile acids $(19.29 \pm$ 3.41 vs. $16.21 \pm 3.68 \mathrm{mg} /$ day, $\mathrm{p}<0.05)$. The two LABtreated groups had similar amount of fecal cholesterol content but the L.9-41-A group had more fecal bile acids $(\mathrm{p}<0.05)$ than the L.M1-16 group.

\section{Visceral organ and WAT weight}

Visceral organ and WAT weight were shown in Table 2. The rats in the control group had the lowest liver and WAT weight. The rats in the L.9-41-A and L.M1-16 groups had significantly lower liver weight than the rats in the model group $(\mathrm{p}<0.05)$. There were no significant differences in the weight of spleens or kidneys among the four groups. Control group had the lowest WAT weight in all four gourps. Compared with the model group, WAT weights extracted from the rats of L.9-41A group was dramatically reduced $(\mathrm{p}<0.05)$, while the L.M1-16 group rats only showed a reduction in the weight of the epididymal fat pad $(\mathrm{p}<0.05)$. Rats of the L.9-41-A group also showed smaller weight of mesenteric and perirenal fat pad than rats of the L.M1-16 group.

\section{Hepatic lipid deposition in the four groups}

The LAB-treated rats exhibited an overall normal gross liver appearance. Liver lipid deposition was evaluated in a semiquantitative manner. As shown in Figure 2, no fatty vacuolization was found in the control group. The 

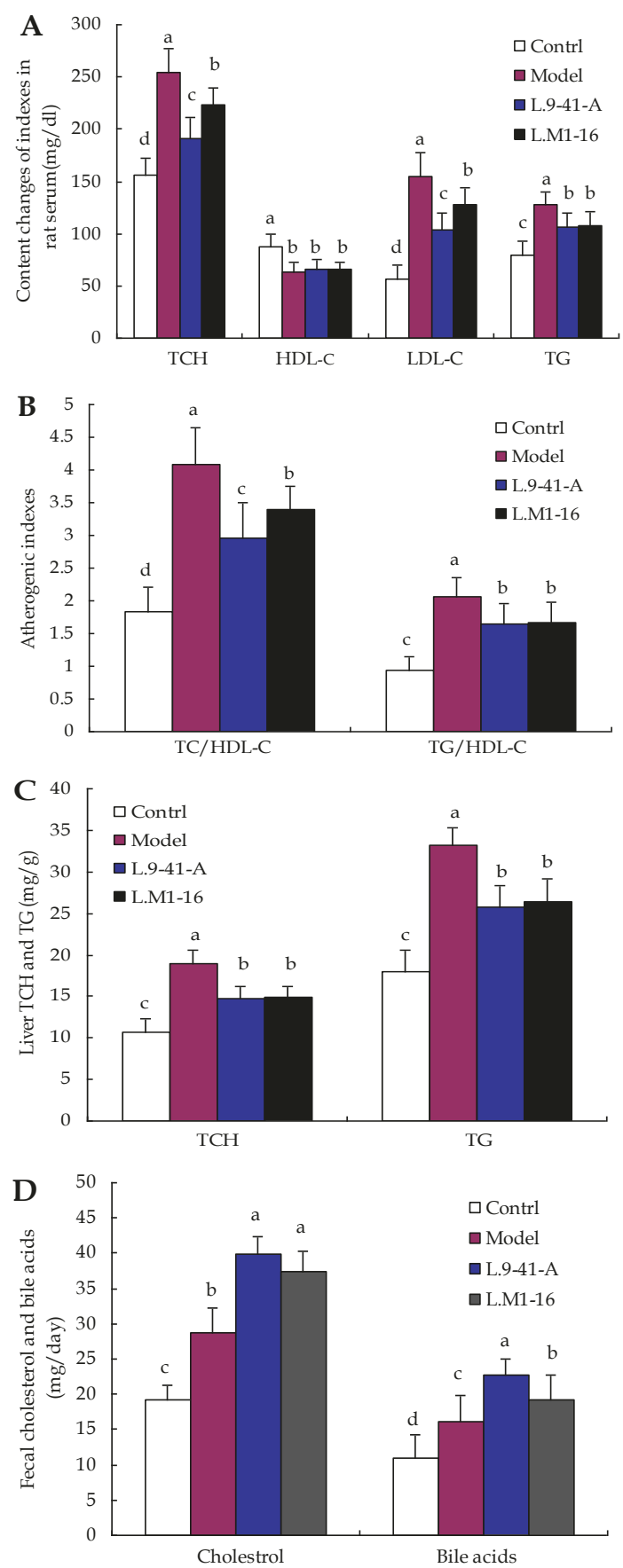

Figure 1 Effects of the two LAB strains on lipid metabolism in rats fed a high-cholesterol diet. A: Serum TCH, HDL-C, LDL-C and TG contents; B: Atherogenic indexes of the rats; C: Liver TCH and TG contents of the rats; D: Fecal total cholesterol and bile acid content. The data are shown as the mean \pm standard deviation $(n=$ 10), ${ }^{a, b, c, d}$ Mean values in each panel with different superscript letters differ significantly $(p<0.05)$.
Table 2 Effects of $L$. plantarum 9-41-A and L. fermentum M1-16 on organ and WAT weight in rats

\begin{tabular}{ccccc}
\hline Weight index(g) & \multicolumn{4}{c}{ Group (mean \pm SD, $\mathbf{n}=10$ per group) } \\
\cline { 2 - 5 } & Control & Model & L.9-41-A & L.M1-16 \\
\hline Liver & $9.5^{\mathrm{c}} \pm 0.8$ & $12.5^{\mathrm{a}} \pm 1.2$ & $10.3^{\mathrm{b}} \pm 1.1$ & $10.7^{\mathrm{b}} \pm 1.4$ \\
Spleen & $0.6 \pm 0.1$ & $0.6 \pm 0.1$ & $0.6 \pm 0.1$ & $0.6 \pm 0.1$ \\
Kidney & $1.7 \pm 0.1$ & $1.7 \pm 0.1$ & $1.7 \pm 0.1$ & $1.7 \pm 0.1$ \\
MFP & $4.0^{\mathrm{b}} \pm 0.4$ & $5.0^{\mathrm{a}} \pm 0.8$ & $4.1^{\mathrm{b}} \pm 0.9$ & $4.8^{\mathrm{a}} \pm 0.6$ \\
PFP & $3.5^{\mathrm{c}} \pm 0.6$ & $6.2^{\mathrm{a}} \pm 1.8$ & $4.9^{\mathrm{b}} \pm 1.0$ & $6.1^{\mathrm{a}} \pm 1.0$ \\
EFP & $4.0^{\mathrm{c}} \pm 0.4$ & $5.4^{\mathrm{a}} \pm 1.3$ & $4.5^{\mathrm{b}} \pm 1.0$ & $4.9^{\mathrm{b}} \pm 1.2$ \\
\hline
\end{tabular}

Control group: normal diet; model group: high-cholesterol diet; L.9-41-A group: high-cholesterol diet + L. plantarum 9-41-A; L.M1-16 group: highcholesterol diet+ L. fermentum M1-16

MFP, mesenteric fat pad; PFP, perirenal fat pad; EFPs, epididymal fat pad

The data are shown as the mean \pm standard deviation ${ }^{a, b, c}$ Mean values within a row with different superscript letters differ significantly $(p<0.05, n=10)$.

liver tissue in the model group had a moderate degree of vacuolization and increased lipid deposition in the cytoplasm, which was obviously lower in the L.9-41-A and L.M1-16 groups. Hepatocyte steatosis was alleviated in the control, L.9-41-A and L.M1-16 groups compared with the model group by a non-parametric test $(\chi 2=$ 24.118, $\mathrm{p}<0.001$ ) (Figure 2 and Table 3).

Effects of L. plantarum 9-41-A and L. fermentum M1-16 on adipocyte numbers and sizes in the WAT

Morphometric analyses of the WAT were shown in Figure 3 and Table 4 . The perirenal WAT in the model group rats had approximately half the numbers of adipocytes observed in the control and L.9-41-A groups. The L.M1-16 group had more adipocytes under each field of view than the model group, but the number was less than the control and L.9-41-A groups $(\mathrm{p}<0.05)$. The average adipocyte size in the model group was significantly $(\mathrm{p}<0.05)$ larger than that in the control and L.9-41-A groups. The L.M1-16 group showed a smaller adipocyte size than that in the model group, although this was not significant.

\section{Microbial populations}

Figure 4 showed the effects of the different diets and $\mathrm{LAB}$ supplementation on the rat intestinal bacteria flora. The model group showed the highest $(\mathrm{p}<0.05)$ total E. coli content (5.7 log CFU/g), followed by the L.9-41A group (4.9 log CFU/g), L.M1-16 group (4.8 log CFU/ g) and control group (4.0 log CFU/g). There were no significant differences in the Enterococcus faecalis colonies among the four groups. Compared with the model group (6.5 log CFU/g for Lactobacillus and $7.2 \mathrm{log}$ CFU/g for Bifidobacterium), significant increases ( $\mathrm{p}<$ 

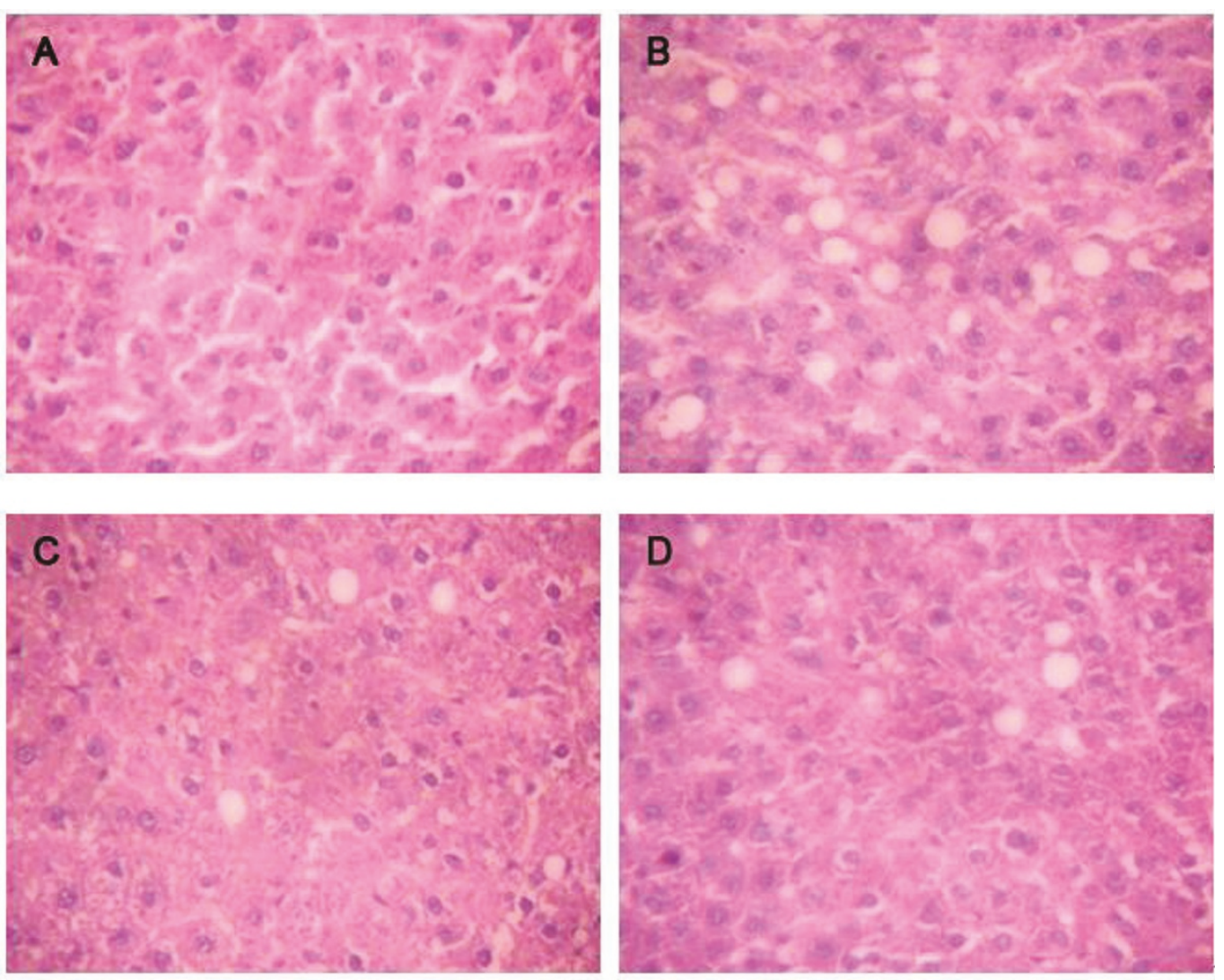

Figure 2 Histology of liver steatosis in the four groups. A: normal diet; B: high-cholesterol diet; C: high-cholesterol diet + L. plantarum 9-41A; D: high-cholesterol diet+ L. fermentum M1-16. All the photomicrographs show HE staining (original magnification $\times 400$ ).

0.05) in the Lactobacillus and Bifidobacterium colonies were observed in the cecal samples from the control group (7.5 log CFU/g for Lactobacillus and $8.1 \mathrm{log}$ CFU/g for Bifidobacterium), L.9-41-A group (8.1 log $\mathrm{CFU} / \mathrm{g}$ for Lactobacillus and $8.0 \mathrm{log}$ CFU/g for Bifidobacterium) and L.M1-16 group (7.9 log CFU/g for Lactobacillus and $8.0 \mathrm{log}$ CFU/g for Bifidobacterium). There were no significant differences $(\mathrm{p}>0.05)$ in the

Table 3 Degrees of liver lipid deposition

\begin{tabular}{ccccc}
\hline Group & \multicolumn{4}{c}{ Degree of fatty deposition* $(\mathbf{n}=\mathbf{1 0}$ per group) } \\
\cline { 2 - 5 } & - & + & ++ & +++ \\
\hline Control & 10 & 0 & 0 & 0 \\
Model & 0 & 6 & 4 & 0 \\
L.9-41-A & 8 & 2 & 0 & 0 \\
L.M1-16 & 7 & 2 & 1 & 0 \\
\hline
\end{tabular}

Control group: normal diet; model group: high-cholesterol diet; L.9-41-A group: high-cholesterol diet + L. plantarum 9-41-A; L.M1-16 group: highcholesterol diet+ L. fermentum M1-16.

*Standard of classification and score for the degree of lipid deposition: +, mild fatty degeneration, fatty hepatocytes occupy $30-50 \%$ of the hepatic parenchyma; ++, moderate fatty degeneration, fatty hepatocytes occupy 50$75 \%$ of the hepatic parenchyma; +++ , severe fatty degeneration, fatty hepatocytes occupy $>75 \%$ of the hepatic parenchyma. number of Lactobacillus and Bifidobacterium colonies between the L.9-41-A group and the L.M1-16 group, and the two groups had more Lactobacillus colonies than the control group $(\mathrm{p}<0.05)$.

\section{Fecal water content}

Fecal water content data collected in the middle of the third and sixth weeks was shown in Figure 5. The fecal water content showed no significant differences in the third week among the four groups (range, $50-55 \%$, p > $0.05)$. However, at the end of the feeding period, there was a higher fecal moisture content in the L.9-41-A (63\%) and L.M1-16 (62\%) groups than in the control $(52 \%)$ and model (53\%) groups.

\section{Discussion}

Elevated serum cholesterol and LDL-C are strongly associated with increasing CVD risks. Recent reports demonstrating the hypocholesterolemic effects of probiotics have led to increased interest in this treatment modality, which is less expensive and could be considered a "natural health remedy". We tested two fecal-derived strains of $\mathrm{LAB}$ and found the hypocholesterolemic effects 

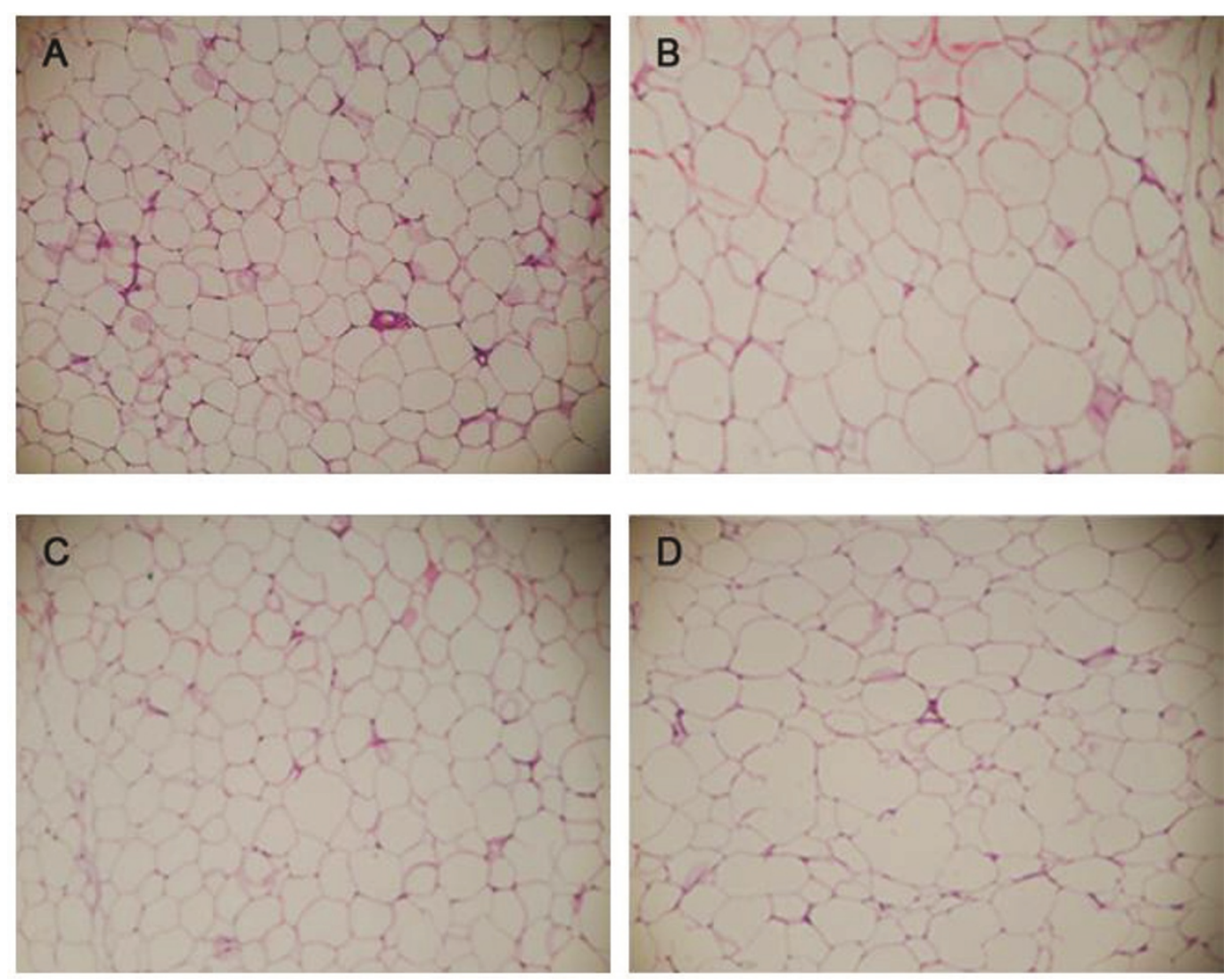

Figure 3 Adipocytes in WAT sections in the four groups. A: normal diet; B:high-cholesterol diet; C: high-cholesterol diet + L. plantarum 9-41A; D: high-cholesterol diet + L. fermentum M1-16. All the photomicrographs show HE staining (original magnification $\times 400$ ).

in vivo. Supplementation with either of the two strains was effective in reducing serum cholesterol, LDL-C and TG concentrations in rats compared to those fed the same high-cholesterol diet but without LAB supplementation. In particular, these effects were more evident with L. plantarum 9-41-A (TCH, TG and liver cholesterol reduced by $25.3 \%, 16.9 \%$ and $22.3 \%$, respectively). Our results confirm those described in previous reports $[6,21,25]$. However, there are also differing reports for Lactobacillus rhamnosus, L. fermentum and Lactobacillus acidophilus $[19,20,26]$. These conflicting findings could be explained by confounding variables, such as different sources and properties of LAB strains, varying clinical characteristics of the subjects, LAB doses, and lengths of treatment[21,27].

LDL-C is the main component of serum cholesterol. Therefore, lowering of the LDL-C level may be an important factor for reducing serum total cholesterol. Our two LAB strains reduced the higher serum LDL-C concentrations in rats caused by the high-cholesterol diet compared with the model group. Park et al. [28] had reported that the hepatic LDL-R mRNA expression of rats fed on either a normal diet or a high-cholesterol diet increased with L. acidophilus ATCC 43121 supplementation and

Table 4 Adipocyte number and size in the WAT under each field ( $\mathrm{n}=10$ per group)

\begin{tabular}{ccccc}
\hline Group & Number/HP & Major axis $(\mu \mathrm{m})$ & Minor axis $(\mu \mathrm{m})$ & Diameter $(\mu \mathrm{m})$ \\
\hline Control & $56.4^{\mathrm{a}} \pm 4.2$ & $36.6^{\mathrm{b}} \pm 3.6$ & $31.5^{\mathrm{b}} \pm 3.2$ & $33.8^{\mathrm{b}} \pm 3.3$ \\
Model & $27.5^{\mathrm{c}} \pm 4.3$ & $55.2^{\mathrm{a}} \pm 4.4$ & $49.19^{\mathrm{a}} \pm 5.6$ & $52.2^{\mathrm{a}} \pm 5.0$ \\
L.9-41-A & $50.3^{\mathrm{a}} \pm 5.7$ & $39.4^{\mathrm{b}} \pm 3.9$ & $36.7^{\mathrm{b}} \pm 2.3$ & $38.0^{\mathrm{b}} \pm 3.0$ \\
L.M1-16 & $39.1^{\mathrm{b}} \pm 6.3$ & $51.9^{\mathrm{a}} \pm 7.3$ & $43.75^{\mathrm{a}} \pm 5.9$ & $47.8^{\mathrm{a}} \pm 6.6$ \\
\hline
\end{tabular}

Control group: normal diet; model group: high-cholesterol diet; L.9-41-A group: high-cholesterol diet + L. plantarum 9-41-A; L.M1-16 group: high-cholesterol diet + L. fermentum M1-16.

Diameter $=($ major axis + minor axis $) / 2$.

The data are shown as the mean \pm standard deviation. ${ }^{a, b, c}$ Mean values within a column with different superscript letters differ significantly $(p<0.05, \mathrm{n}=10$ ). 


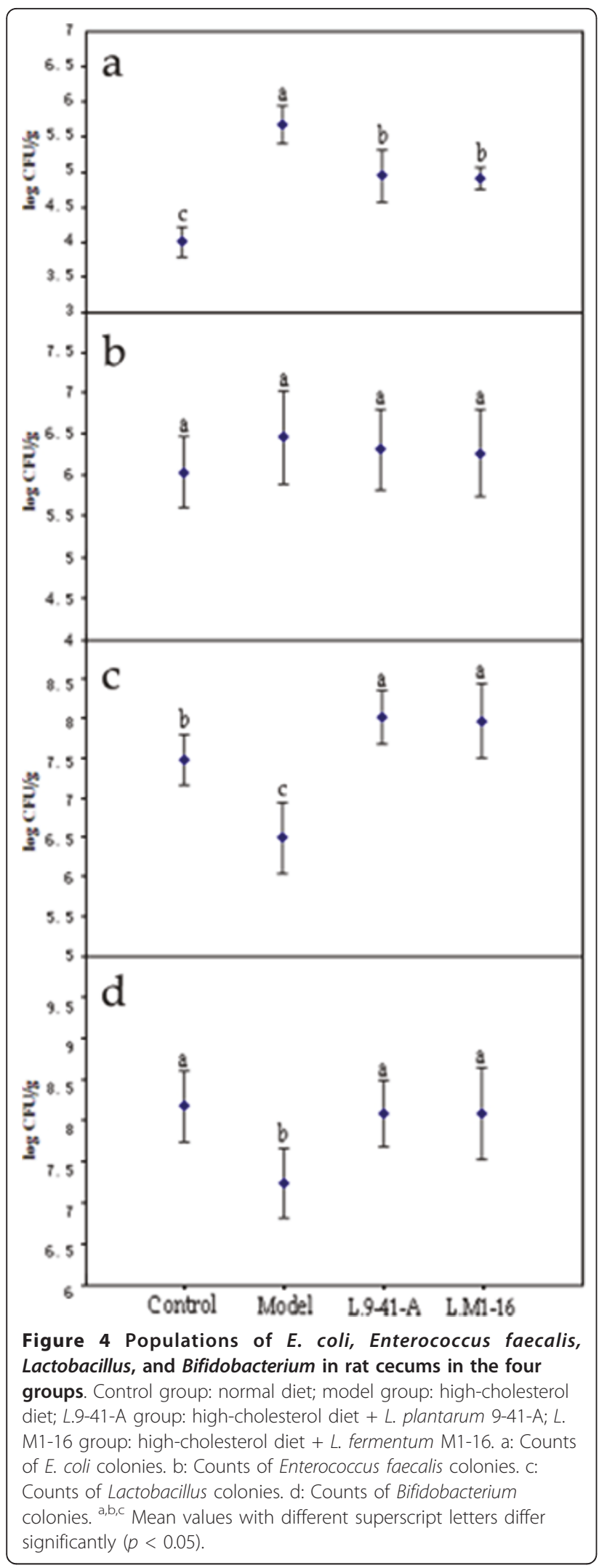

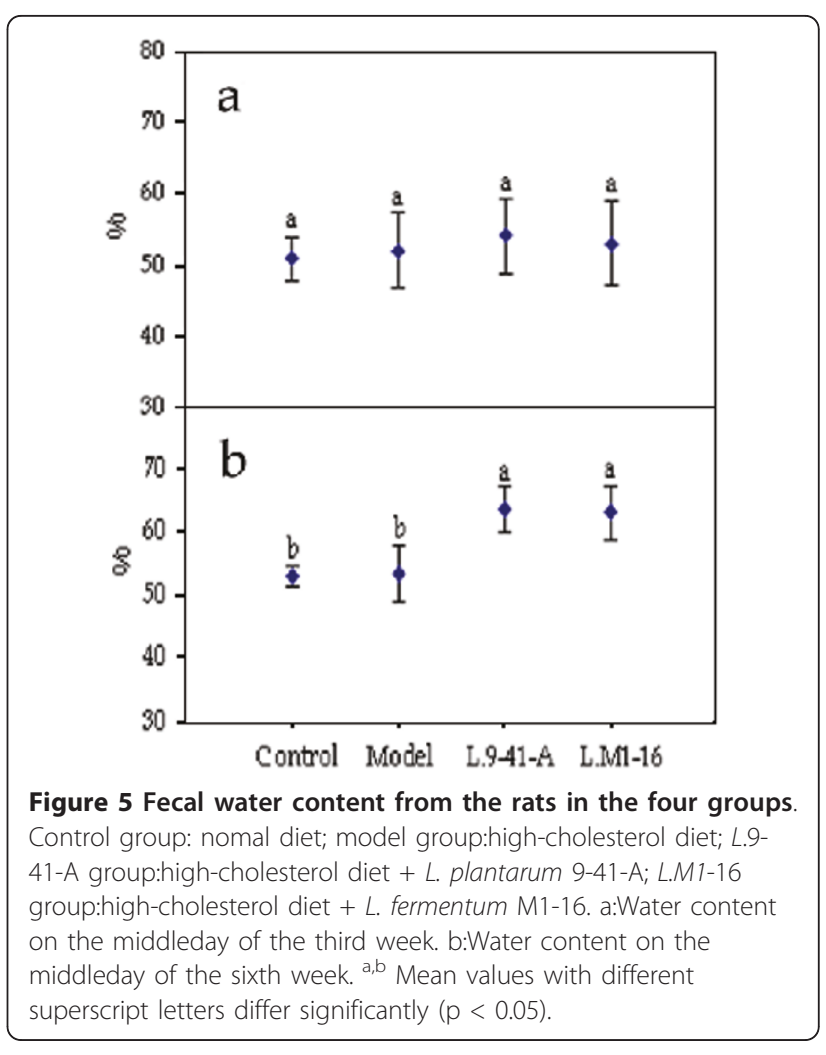

the serum LDL-C level reduced accordingly, but by what means the LAB affect the hepatic LDL-R mRNA expression needs to be investigated furtherly. The two strains did not appear to affect HDL-C concentrations. Ibrahim et al. [29] and St-Onge et al. [30] reported similar results about the influence of fermented milk on the HDL-C level in rats or humans. However, Hashimoto et al. [31] found that a diet containing Lactobacillus casei TMC 0409 could increase the concentration of HDL-C in rats. Shin et al. [32] also recently reported that the serum HDL-C levels in rats were increased by sonication-killed Bifidobacterium longum. A convincing explanation for these controversial findings remains unclear, although different properties of the bacterial strains could be considered. We also found that the TCH/HDL-C and TG/ HDL-C ratios, which are highly independent predictors of CVD [33,34], were reduced in rats supplemented with LAB. Further studies are necessary to confirm these observations, and thereby more definitively predict whether LAB supplementation does reduce the risk of CVD.

As expected, we confirmed that the high-cholesterol diet increased hepatic cholesterol and TG content in rats. Rats supplemented with L. plantarum 9-41-A and L. fermentum M1-16 displayed significant reductions in hepatic cholesterol and TG levels and lipid deposition. 
These findings demonstrated that the serum cholesterol and TG levles in LAB-treated rats were actually reduced, rather than merely being redistributed from the blood to the liver. The lower level of hepatic cholesterol and TG content could decrease the conversion of IDL to LDL-C particles, and result in the decrease of serum LDL-C concentration, that might be another reason for the effects of our two strains to lower LDL-C level.

It has been reported that certain probiotic strains could enhance fecal elimination of bile acids and this may alter the cholesterol synthesis pathways and resulted in a decrease of serum cholesterol concentration [28]. We found more bile acids in the feces of the LAB-treated rats, especially in those rats in the L.9-41-A group. Therefore, one possible mechanism for how our two strains lowered the cholesterol levels may be that more bile acids in the enterohepatic circulation were deconjugated and precipitated, and excreted from the feces, resulting in reduced serum cholesterol concentrations. This may partly explain that why rats in the L.941-A group had lower serum cholesterol level than rats in the L.M1-16 group. In addition, more fecal cholesterol was detected in the L.9-41-A and L.M1-16 group rats. LAB may assimilate dietary cholesterol by incorporating it into their cellular membranes or cell walls, and then via fecal excretion [35]. Thus, LAB strains with hypocholesterolemic properties may lower serum cholesterol in multiple ways.

Generally, a high-cholesterol diet could increase the body weight. To the best of our knowledge, only a few studies have reported a weight-lowering effect of LAB besides a hypocholesterolemic ability. We observed significant weight loss $(\mathrm{p}<0.05)$ in the rats supplemented with L. plantarum 9-41-A compared with the model group rats, and decreased liver and adipose tissue weight contributed to this finding. In addition, adipocytes of the mesenteric WAT in the L.9-41-A rats were smaller than those in the model group rats. Some bacterial strains are considered to have preventive effects on excessive body weight gain[22,36,37]. Fat digestion and absorption in the small intestine may be affected by the gut microbiota. Hamad et al. [36] reported a reduction in the adipocyte size of rats treated with fermented skimmed milk containing Lactobacillus gasseri SBT2055. The authors therefore considered that the skimmed milk fermented by L. gasseri SBT2055 would reduce fat storage through the inhibition of dietary fat absorption. Increased bile acid excretion in the feces could elevate bile acid synthesis, and this process may also contribute to the observed weight-lowering effect. Ikemoto et al. [38] found that cholate could inhibit diet-induced obesity by decreasing acyl-CoA synthetase mRNA expression. Watanabe et al. [39] also reported that bile acids could induce energy expenditure. Nevertheless, additional research on the mechanisms behind these actions is required.

Gut microbiota distortions may potentially contribute to a wide range of diseases. Effective interventions in the gut microbiota composition with probiotic supplementation may improve health and prevent the onset of certain diseases[40]. Analyses of the rat intestinal microflora demonstrated that E. coli was increased while Lactobacillus and Bifidobacterium were decreased in the model group, implying that the high-cholesterol diet may have interfered with the intestinal microbiota. Stepankova et al. [41] found that absence of gut microbiota (germ-free conditions) accelerates the atherosclerosis in apoE-deficient mice, which also indicates the existance of the relationship between intestinal microbiota and serum cholesterol levels. Here we presume that LAB may have successfully ameliorated the intestinal microbiota disorder induced by a high-cholesterol diet and exerting a beneficial effect that influenced lipid profiles. The exact mechanism in vivo need be studied further to verify our presumption. Certain LAB strains have been shown to inhibit the proliferation of pathogenic bacteria $[42,43]$. In our study, L. plantarum 9-41-A and L. fermentum M1-16 inhibited the growth of E. coli; in addition, the numbers of Lactobacillus and Bifidobacterium were significantly higher in the LAB-treated rats, suggesting that the two strains used can successfully tolerate gastric acid and bile salts, then benefit the proliferation of Lactobacillus and Bifidobacterium which are considered benefical to humans. Imbalances in the gut microflora could be considered to be environmental factors involved in the development of obesity and its associated metabolic disorders [44-46]. Modulation of the intestinal microbiota by supplementation with certain LAB strains may lead to body weight reduction.

By detecting the fecal water content of all rats we found that L. plantarum 9-41-A and L. fermentum M116 increased the fecal water content in rats. Since the fecal water content can be used as an index of fecal elimination, our observations suggest that these two LAB strains have laxative potential and may stimulate bowel movements. Consequently, the transit time for cholesterol absorption in the intestine might also be reduced. This could be another explanation for the cholesterol-lowering ability of LAB strains.

Our study had some limitations, we only used SpragueDawley rats, and therefore, we cannot deny that the effects of LAB on cholesterol may be specific for this particular strain, and not general. So before a definite conclusion about the hypocholesterolemic effects of our lactobacilli can be reached, at least one to two more animal models require testing. Besides, Chinese people are mainly on a eastern dietaty pattern which includes wheat, rice, vegetables and fruits. Since diet can play a role in the composition and metabolic characteristics of microbiota residented 
in the mammalian gastrointestinal tract [47], the two strains in our present study which were isolated from the stool of healthy adult Chinese volunteers might be more suitable for the future application on Asian people who are mainly on a eastern dietaty pattern, more intensive researchs should be conducted to prove this presumption.

\section{Conclusions}

In summary, fecal-derived $L$. plantarum 9-41-A and $L$. fermentum M1-16 exerted significant hypocholesterolemic effect on Sprague-Dawley rats fed a high-cholesterol diet, with the former strain trending towards more significant reductive effects. Accordingly, the L.9-41-A strain resulted in more significant body weight loss and lower fat storage. The two strains are also able to contribute to a healthier intestinal microbial banlance. These findings indicate that the effects of LAB on lipid metabolism may differ among strains, and that a search for more potent and better bacterial strains was meaningful. The L. plantarum 9-41-A strain may represent a potential therapeutic agent for controlling hyperlipidemia and lessening excessive body weight gain. The mechanisms behind these effects are likely to be diverse and will require further investigations in vivo.

\section{List of abbreviations}

LAB: Lactic acid bacteria; L.9-41-A: Lactobacillus plantarum 9-41-A; L.M1-16: Lactobacillus fermentum M1-16; E. coli: Escherichia coli; CVD: cardiovascular diseases; WHO: World Health Organization; CFU: Colony-Forming Units; WAT: white adipose tissue; HE: hematoxylin-eosin; SD: standard deviations.

\section{Acknowledgements}

This work was supported by a grant from the National Natural Science Foundation of China (No.81070295).

\section{Author details \\ 'Department of Gastroenterology, Xiangya Second Hospital, Central South University, Changsha 410011, Hunan Province, PR China. ${ }^{2}$ Department of Gastroenterology, Nanhua Second Hospital, Nanhua University, Hengyang 421001, Hunan Province, China. ${ }^{3}$ Department of Gastroenterology, Nanhua Hospital, Nanhua University, Hengyang 421002, Hunan Province, China. ${ }^{4}$ State Key Laboratory of Medical Genetics, Central South University, Changsha 410011, Hunan Province, China.}

\section{Authors' contributions}

$X N$, WXH and LFG designed the study; XN, YYN, ZX, YJW, CY, WZG, FN and TY were involved in experiment conduction and analysis; $X N, W X H, L X W$, WCL and LFG performed data interpretation, presentation and wrote the manuscript; LXW and LFG provided significant academic advice and consultation. All authors read and approved the final manuscript.

\section{Competing interests}

The authors declare that they have no competing interests.

Received: 6 March 2011 Accepted: 3 July 2011 Published: 3 July 2011

\section{References}

1. WHO: Cardiovascular Disease; Fact sheet №317, Geneva, Switzerland. 2009 [http://www.who.int/mediacentre/factsheets/fs317/en/], (accessed on 5 July 2010).

2. Manson JE, Tosteson H, Ridker PM, Satterfield S, Hebert P, O'Connor GT, Buring JE, Hennekens $\mathrm{CH}$ : The primary prevention of myocardial infarction. N Engl J Med 1992, 326(21):1406-1416.
3. Mann GV, Spoerry A: Studies of a surfactant and cholesteremia in the Maasai. Am J Clin Nutr 1974, 27:464-469.

4. Fukushima M, Nakano M: Effects of a mixture of organisms, Lactobacillus acidophilus or Streptococcus faecalis on cholesterol metabolism in rats fed on a fat- and cholesterol-enriched diet. Br J Nutr 1996, 76(6):857-867.

5. Gilliland SE, Nelson CR, Maxwell C: Assimilation of cholesterol by Lactobacillus acidophilus. Appl Environ Microbiol 1985, 49(2):377-381.

6. Nguyen TD, Kang JH, Lee MS: Characterization of Lactobacillus plantarum PH04, a potential probiotic bacterium with cholesterol-lowering effects. Int J Food Microbiol 2007, 113(3):358-361.

7. Agerbaek M, Gerdes LU, Richelsen B: Hypocholesterolaemic effect of a new fermented milk product in healthy middle-aged men. Eur J Clin Nutr 1995, 49(5):346-352.

8. Anderson JW, Gilliland SE: Effect of fermented milk (yogurt) containing Lactobacillus acidophilus L1 on serum cholesterol in hypercholesterolemic humans. J Am Coll Nutr 1999, 18(1):43-50.

9. Keim NL, Marlett JA, Amundson CH: The cholesterolemic effect of skim milk in young men consuming controlled diets. Nutr Res 1981, 1(5):429-442.

10. Xiao JZ, Kondo S, Takahashi N, Miyaji K, Oshida K, Hiramatsu A, Iwatsuki K, Kokubo S, Hosono A: Effects of milk products fermented by Bifidobacterium longum on blood lipids in rats and healthy adult male volunteers. J Dainy Sci 2003, 86(7):2452-2461.

11. Pigeon RM, Cuesta EP, Gililliand SE: Binding of free bile acids by cells of yogurt starter culture bacteria. J Dairy Sci 2002, 85(11):2705-2710.

12. Pereira DI, Gibson GR: Cholesterol assimilation by lactic acid bacteria and bifidobacteria isolated from the human gut. Appl Environ Microbiol 2002, 68(9):4689-4693.

13. Liong MT, Shah NP: Acid and bile tolerance and cholesterol removal ability of lactobacilli strains. J Dairy Sci 2005, 88(1):55-66.

14. Lye H-S, Rahmat-Ali GR, Liong M-T: Mechanisms of cholesterol removal by lactobacilli under conditions that mimic the human gastrointestinal tract. International Dairy Journal 2010, 20(3):169-175.

15. Lye HS, Rusul G, Liong MT: Removal of cholesterol by lactobacilli via incorporation and conversion to coprostanol. J Dairy Sci 2010, 93(4):1383-1392.

16. Cheeke PR: Actual and potential applications of Yucca schidigera and Quillaja saponaria saponins in human and animal nutrition. J Anim Sci 2000, 77:1-10.

17. Trautwein EA, Rieckhoff D, Erbersdobler HF: Dietary inulin lowers plasma cholesterol and triacylglycerol and alters biliary bile acid profile in hamsters. J Nutr 1998, 128(11):1937-1943.

18. Begley M, Hill C, Gahan CG: Bile salt hydrolase activity in probiotics. Appl Environ Microbiol 2006, 72(3):1729-1738.

19. Hatakka K, Mutanen M, Holma R, Saxelin M, Korpela R: Lactobacillus rhamnosus LC705 together with Propionibacterium freudenreichii ssp shermanii JS administered in capsules is ineffective in lowering serum lipids. J Am Coll Nutr 2008, 27(4):441-447.

20. Simons LA, Amansec SG, Conway P: Effect of Lactobacillus fermentum on serum lipids in subjects with elevated serum cholesterol. Nutr Metab Cardiovasc Dis 2006, 16(8):531-535.

21. Wang $Y, X u N, X i$ A, Ahmed Z, Zhang B, Bai X: Effects of Lactobacillus plantarum MA2 isolated from Tibet kefir on lipid metabolism and intestinal microflora of rats fed on high-cholesterol diet. Appl Microbiol Biotechnol 2009, 84(2):341-347.

22. Yin YN, Yu QF, Fu N, Liu XW, Lu FG: Effects of four Bifidobacteria on obesity in high-fat diet induced rats. World J Gastroenterol 2010, 16(27):3394-3401.

23. Tokunaga T, Oku T, Hosoya N: Influence of chronic intake of new sweetener fructooligosaccharide (Neosugar) on growth and gastrointestinal function of the rat. J Nutr Sci Vitaminol 1986, 32:111-121.

24. Minoura H, Takeshita S, Kimura C, Hirosumi J, Takakura S, Kawamura I, Seki J, Manda T, Mutoh S: Mechanism by which a novel non-thiazolidinedione peroxisome proliferator-activated receptor gamma agonist, FK614, ameliorates insulin resistance in Zucker fatty rats. Diabetes Obes Metab 2007, 9(3):369-378.

25. Chiu CH, Lu TY, Tseng YY, Pan TM: The effects of Lactobacillus-fermented milk on lipid metabolism in hamsters fed on high-cholesterol diet. Appl Microbiol Biotechnol 2006, 71(2):238-245.

26. Lewis SJ, Burmeister S: A double-blind placebo-controlled study of the effects of Lactobacillus acidophilus on plasma lipids. Eur J Clin Nutr 2005, 59(6):776-780. 
27. Liong MT: Probiotics: a critical review of their potential role as antihypertensive, immune modulators, hypocholesterolemics and perimenopausal treatments. Nutr Rev 2007, 65:1-13.

28. Park YH, Kim JG, Shin YW, Kim SH, Whang KY: Effect of dietary inclusion of Lactobacillus acidophilus ATCC 43121 on cholesterol metabolism in rats. J Microbiol Biotechnol 2007, 17(4):655-662.

29. Ibrahim A, El-sayed E, El-zeini H, HM S: The hypocholesterolemic effect of milk yogurt and soy-yogurt containing bifidobacteria in rats fed on a cholesterol-enriched diet. Int Dairy J 2005, 15:37-44.

30. St-Onge MP, Farnworth ER, Savard T, Chabot D, Mafu A, Jones PJ: Kefir consumption does not alter plasma lipid levels or cholesterol fractional synthesis rates relative to milk in hyperlipidemic men:a randomized controlled trial. BMC Complement Altern Med 2002, 2:1-7.

31. Hashimoto $H$, Yamazaki $K$, He F, Kawase M, Hosoda M, Hosono A: Hypocholesterolemic effects of Lactobacillus casei subsp. casei TMC 0409 strain observed in rats [Rattus norvegicus] fed cholesterol contained diets. Anim Sci J 1999, 70(2):90-97.

32. Shin HS, Park SY, Lee do K, Kim SA, An HM, Kim JR, Kim MJ, Cha MG, Lee SW, Kim KJ, Lee KO, Ha NJ: Hypocholesterolemic effect of sonicationkilled Bifidobacterium longum isolated from healthy adult Koreans in high cholesterol fed rats. Arch Pharm Res 2010, 33(9):1425-1431.

33. da Luz PL, Favarato D, Faria-Neto JR Jr, Lemos P, Chagas AC: High ratio of triglycerides to $\mathrm{HDL}$-cholesterol predicts extensive coronary disease. Clinics (Sao Paulo) 2008, 63(4):427-432.

34. Lampka M, Grabczewska Z, Jendryczka-Mackiewicz E, Holynska-Iwan I, Sukiennik A, Kubica J, Halota W, Tyrakowski T: Circulating endothelial cells in coronary artery disease. Kardiol Pol 2010, 68(10):1100-1105.

35. Liong MT, Shah NP: Effects of a Lactobacillus casei synbiotic on serum lipoprotein, intestinal microflora, and organic acids in rats. J Dairy Sci 2006, 89(5):1390-1399.

36. Hamad EM, Sato M, Uzu K, Yoshida T, Higashi S, Kawakami H, Kadooka Y, Matsuyama H, Abd El-Gawad IA, Imaizumi K: Milk fermented by Lactobacillus gasseri SBT2055 influences adipocyte size via inhibition of dietary fat absorption in Zucker rats. Br J Nutr 2009, 101(5):716-724.

37. Kang JH, Yun Sl, Park HO: Effects of Lactobacillus gasseri BNR17 on body weight and adipose tissue mass in diet-induced overweight rats. $J$ Microbiol 2010, 48(5):712-714.

38. Ikemoto S, Takahashi M, Tsunoda N, Maruyama K, Itakura H, Kawanaka K, Tabata I, Higuchi M, Tange T, Yamamoto TT, Ezaki O: Cholate inhibits highfat diet-induced hyperglycemia and obesity with acyl-CoA synthetase mRNA decrease. Am J Physiol 1997, 273(1 Pt 1):E37-45.

39. Watanabe M, Houten SM, Mataki C, Christoffolete MA, Kim BW, Sato H, Messaddeq N, Harney JW, Ezaki O, Kodama T, Schoonjans K, Bianco AC, Auwerx J: Bile acids induce energy expenditure by promoting intracellular thyroid hormone activation. Nature 2006, 439(7075):484-489.

40. Mai V, Draganov PV: Recent advances and remaining gaps in our knowledge of associations between gut microbiota and human health. World J Gastroenterol 2009, 15(1):81-85.

41. Stepankova R, Tonar Z, Bartova J, Nedorost L, Rossman P, Poledne R, Schwarzer M, Tlaskalova-Hogenova H: Absence of microbiota (germ-free conditions) accelerates the atherosclerosis in ApoE-deficient mice fed standard low cholesterol diet. J Atheroscler Thromb 2010, 17(8):796-804.

42. Fuller R: Probiotics in man and animals. J App/ Bacteriol 1989, 66(5):365-378

43. Van Winsen RL, Keuzenkamp D, Urlings BA, Lipman L, Snijders JA, Verheijden $\mathrm{JH}$, van Knapen F: Effect of fermented feed on shedding of Enterobacteriaceae by fattening pigs. Vet Microbiol 2002, 87(3):267-276.

44. Cani PD, Delzenne NM: Interplay between obesity and associated metabolic disorders: new insights into the gut microbiota. Curr Opin Pharmacol 2009, 9(6):737-743.

45. Scarpellini E, Campanale M, Leone D, Purchiaroni F, Vitale G, Lauritano EC, Gasbarrini A: Gut microbiota and obesity. Intern Emerg Med 2010, 5(Suppl 1):S53-56.

46. Waldram A, Holmes E, Wang Y, Rantalainen M, Wilson ID, Tuohy KM, McCartney AL, Gibson GR, Nicholson JK: Top-down systems biology modeling of host metabotype-microbiome associations in obese rodents. J Proteome Res 2009, 8(5):2361-2375.

47. Buddington RK, Sangild PT: COMPANION ANIMALS SYMPOSIUM: Development of the mammalian gastrointestinal tract, the resident microbiota, and the role of diet in early life. J Anim Sci 2011, 89(5):1506-1519.

\section{Pre-publication history}

The pre-publication history for this paper can be accessed here: http://www.biomedcentral.com/1472-6882/11/53/prepub

\section{doi:10.1186/1472-6882-11-53}

Cite this article as: Xie et al.: Effects of two Lactobacillus strains on lipid metabolism and intestinal microflora in rats fed a high-cholesterol diet. BMC Complementary and Alternative Medicine 2011 11:53.

\section{Submit your next manuscript to BioMed Central and take full advantage of:}

- Convenient online submission

- Thorough peer review

- No space constraints or color figure charges

- Immediate publication on acceptance

- Inclusion in PubMed, CAS, Scopus and Google Scholar

- Research which is freely available for redistribution

Submit your manuscript at www.biomedcentral.com/submit
Biomed Central 